\title{
Three-dimensional quasiperiodic torsional flows in rotating spherical fluids at very low Prandtl numbers
}

\author{
J. Sánchez Umbría* and M. Net ${ }^{\dagger}$ \\ Physics Department, Universitat Politècnica de Catalunya, \\ Jordi Girona Salgado 1-3, Campus Nord, \\ Mòdul B4, 08034 Barcelona, Spain
}

(Dated: October 3, 2021)

\begin{abstract}
The aim of this study is to determine through numerical simulations the extent and robustness of the three-dimensional torsional dynamics of the thermal convection in rotating spherical fluids at very low Prandtl numbers. It is known that the kinetic energy of the periodic axisymmetric flows propagates latitudinally on the surface of the sphere. Here it is shown that when the axisymmetry is broken at a secondary Hopf bifurcation, the flow starts to drift in the azimuthal direction giving rise to a quasiperiodic motion that propagates the energy in latitude and longitude. The double direction of propagation gives rise to a meandering path of the kinetic energy, which is still concentrated on the surface, but highly localized. Several new stable states of convection with different symmetries have been identified in a large range of Rayleigh numbers, all of them retaining the torsional motion of the basic velocity field. Particular attention is paid to their dependence on the Rayleigh number, and on the values of the frequencies, of the mean zonal flow, and of the kinetic energy of the fluid.

PACS numbers: 47.15.-x, 47.20.-k
\end{abstract}

Keywords: Thermal convection, Time-dependent flows, Bifurcations, Symmetry breaking

\footnotetext{
* juan.j.sanchez@upc.edu

† marta.net@upc.edu
} 


\section{INTRODUCTION}

The study of the thermal convection in rotating fluid spheres and spherical shells is essential because it affects the dynamics and the generation of magnetic fields of planets and stars. There are several reasons for which it is much less understood at very low Prandtl numbers, Pr, than at moderate and high Pr. On the one hand the experiments with liquid sodium are very expensive, of high technical difficulty and, in addition, it is not easy to visualize directly the patterns of convection at low Rayleigh numbers, Ra. On the other hand the numerical simulations are very expensive. Very small time steps must be taken due to the small period of the oscillations, and the proximity of various branches of solutions lengthen the transients. Despite the difficulties, the experimental and numerical studies have intensified in recent years, reaching low Ekman numbers at the very low Pr of liquid metals, E, [1-4], or taking into account new phenomena such us the precession of the rotating spheres [5], the compressibility of the fluid $[6,7]$, the influence of Robin [8] and fixed-flux [9] boundary conditions for the temperature, or attaining the fully developed turbulence $[10,11]$.

Of particular interest in the context of the present work are the numerical studies $[12,13]$. They have shown that with stress-free and impenetrable boundary conditions, radial gravity, internal heating, and when the ratio of $\operatorname{Pr}$ to $E$, is $\mathcal{O}(10)$, the onset of convection takes place after a Hopf bifurcation that gives rise to periodic axisymmetric flows $(\varphi$-independent in spherical coordinates $(r, \theta, \varphi), \theta$ being the colatitude and $\varphi$ the longitude), of period $T$, nearly antisymmetric with respect to the equator. The antisymmetric component of the velocity field fulfills $\left(v_{r}^{a}, v_{\theta}^{a}, v_{\varphi}^{a}\right)(t, r, \theta, \varphi)=\left(-v_{r}^{a}, v_{\theta}^{a},-v_{\varphi}^{a}\right)\left(t, r, \pi-\theta, \varphi+\varphi_{0}\right)$, and that of the temperature $\mathrm{T}^{a}(t, r, \theta, \varphi)=-\mathrm{T}^{a}\left(t, r, \pi-\theta, \varphi+\varphi_{0}\right), \varphi_{0}$ being any arbitrary azimuthal angle). Moreover the velocity field retains the spatio-temporal symmetry $\left(v_{r}, v_{\theta}, v_{\varphi}\right)(t, r, \theta, \varphi)=$ $\left(v_{r}, v_{\theta}, v_{\varphi}\right)\left(t+T / 2, r, \pi-\theta, \varphi+\varphi_{0}\right)$ and $\mathrm{T}(t, r, \theta, \varphi)=\mathrm{T}\left(t+T / 2, r, \pi-\theta, \varphi+\varphi_{0}\right)$. The flow consists of a meridional (poloidal) vortex, which fills the sphere and reverses its rotation every half period, and an azimuthal motion with opposite velocities in each hemisphere, which also changes its direction but with a phase shift relative to the poloidal field. This type of flow is known as torsional. The periodic axisymmetric solutions are stable in a very narrow range of $\mathrm{Ra}$ [14], and they lose stability to three-dimensional velocity fields. It can seem at first sight that the conditions for the existence of the torsional motion are very 
restrictive to be relevant in geophysics and astrophysics. However, a recent study of the onset of convection in a fluid sphere heated from the inside [15] delimited the bounds of the region of parameters where the first bifurcation from the conduction state leads to a torsional pattern of convection. It was shown that below $E \approx 6 \times 10^{-2}$, and in particular in the limit $E \rightarrow 0$, there is always an interval of Pr in which the first transition is to torsional solutions. The fit of the stability curves is potential, i.e., $E \propto \operatorname{Pr}^{n}$, narrowing when $E \rightarrow 0$. In addition, a second point of interest to study these flows is that the non-periodic torsional velocity fields are able to generate and sustain strong magnetic fields [16].

The three- and two-dimensional dynamics of the torsional convection at low Ra was studied numerically by Kong et al. [14], and Sánchez Umbría and Net [17], respectively. Both simulations found a wave of kinetic energy propagating latitudinally when the nonlinear effects weakly break the exact anti-symmetry with respect to the equator of the eigenfunction. The main aim of the following study is to deep in the influence of the axisymmetric bifurcation on the three-dimensional flows, after a secondary bifurcation on the branch of torsional flows takes place. In this way this work complements that by Kong et al. [14], who describe the dynamics on the branch arising at the second bifurcation from the conduction state with $\operatorname{Pr}=10^{-2}, E=10^{-3}$ and a very small ratio of radius $\eta=0.001$. The convection following that branch is an equatorially symmetric wave of azimuthal wavenumber $m=1$, traveling in the retrograde direction with constant kinetic energy, that bifurcates to a quasiperiodic branch of mixed dynamics.

The remainder of the paper is organized as follows. After the introduction, Sec. II is devoted to introduce the mathematical model. Some resolution tests are presented in Sec. II B. Section III studies the temporal dependence and mean properties of the flow from the onset of convection to, approximately, twice the critical Ra. Section IV describes the dynamics of the quasiperiodic torsional flows, and in Sec. $\mathrm{V}$ the comparison of this dynamics with that found in low-dimensional models is established. Finally, Sec. VI summarizes the main results together with the conclusions of the work.

\section{FORMULATION, NUMERICAL METHODS AND TESTS}

The dynamics arising in internally heated fluid spheres or spherical shells of dimensional internal radius $r_{i}^{*}$, and external, $r_{o}^{*}$, rotating about a symmetry axis with constant angular 


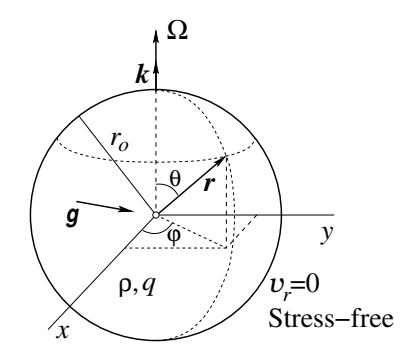

FIG. 1. Geometry and physical settings of the problem. The symbol $\rho$ accounts for the density of the fluid, and $q$ for its internal heating.

velocity $\Omega=\Omega \boldsymbol{k}$, and subject to radial gravity $\boldsymbol{g}=-\gamma \boldsymbol{r}$, can be studied by using the Boussinesq approximation of the mass, momentum and energy equations. In the definition of the gravity acceleration $\gamma$ is a constant (corresponding to a uniform density), and $\boldsymbol{r}$ the position vector. The number of equations can be reduced by writing the velocity field in terms of toroidal, $\Psi$, and poloidal, $\Phi$, potentials as

$$
\boldsymbol{v}=\nabla \times(\Psi \boldsymbol{r})+\nabla \times \nabla \times(\Phi \boldsymbol{r})
$$

In order to emulate the thermal convection in fluid spheres it is a common practice to take wide spherical shells of very small radius ratio $\eta=r_{i}^{*} / r_{o}^{*}$. For instance, $\eta=0.001$ was used in [14]. It was shown that the presence of a very small internal sphere does not affect the supercritical Hopf bifurcation, which maintains the axisymmetry of the conduction state, and only slightly modifies the value of the critical Ra. This approach has been adopted in this study by setting $\eta=0.01$. An scheme of the geometry and physical formulation of the problem is included in Fig. 1.

The nondimensional equations in the rotating frame of reference, written for both potentials and the temperature perturbation from the basic state, $\Theta=\mathrm{T}-\mathrm{T}_{c}$, are

$$
\begin{aligned}
\left(\partial_{t}-\Delta\right) \mathcal{L}_{2} \Psi & =2 E^{-1}(\partial \varphi \Psi-\mathcal{Q} \Phi)-\boldsymbol{r} \cdot \boldsymbol{\nabla} \times(\boldsymbol{\omega} \times \boldsymbol{v}) \\
\left(\partial_{t}-\Delta\right) \mathcal{L}_{2} \Delta \Phi & =2 E^{-1}(\partial \varphi \Delta \Phi+\mathcal{Q} \Psi)-\mathcal{L}_{2} \Theta+\boldsymbol{r} \cdot \boldsymbol{\nabla} \times \boldsymbol{\nabla} \times(\boldsymbol{\omega} \times \boldsymbol{v}) \\
\left(\operatorname{Pr} \partial_{t}-\Delta\right) \Theta & =\operatorname{Ra} \mathcal{L}_{2} \Phi-\operatorname{Pr}(\boldsymbol{v} \cdot \boldsymbol{\nabla}) \Theta
\end{aligned}
$$

The operators $\mathcal{L}_{2}$ and $\mathcal{Q}$ are defined by $\mathcal{L}_{2} \equiv-r^{2} \Delta+\partial_{r}\left(r^{2} \partial_{r}\right), \mathcal{Q} \equiv r \cos \theta \Delta-\left(\mathcal{L}_{2}+\right.$ $\left.r \partial_{r}\right)\left(\cos \theta \partial_{r}-r^{-1} \sin \theta \partial_{\theta}\right)$. In addition, $\boldsymbol{\omega}=\boldsymbol{\nabla} \times \boldsymbol{v}$ is the vorticity. 
The units employed in Eqs. (2)-(4) are the gap width, $d=r_{o}^{*}-r_{i}^{*}$, for the distance, $\nu^{2} / \gamma \alpha d^{4}$ for the temperature, and $d^{2} / \nu$ for the time. With this scaling Pr, Ra, and $E$ are

$$
\operatorname{Pr}=\frac{\nu}{\kappa}, \quad \operatorname{Ra}=\frac{q \gamma \alpha d^{6}}{3 c_{p} \kappa^{2} \nu}, \quad E=\frac{\nu}{\Omega d^{2}} .
$$

The coefficients $q, c_{p}, \nu$ and $\kappa$ are the rate of internal heat generated per unit mass, the specific heat at constant pressure, the kinematic viscosity and the thermal diffusivity, respectively. The spherical symmetric conduction state is then given by $\boldsymbol{v}=\mathbf{0}$ and $\mathrm{T}_{c}(r)=\mathrm{T}_{0}-(\mathrm{Ra} / 2 \mathrm{Pr}) r^{2}$ (state $S_{0}$ from now on), so it fulfills

$$
\mathrm{T}(t, r, \theta, \varphi)=\mathrm{T}\left(t+t_{0}, r, \theta+\theta_{0}, \varphi+\varphi_{0}\right)
$$

for arbitrary $t_{0}, \theta_{0}$ and $\varphi_{0}$. The centrifugal force is omitted from the formulation because in many self-gravitating bodies the rotational Froude number, $F r=\Omega^{2} R / g$, and the flattening, $f l=\left(R_{\max }-R_{\min }\right) / R_{\max }$, are much less than one. For instance, taking the rotation $\Omega_{\odot}=2.69 \times 10^{-6} \mathrm{~s}^{-1}$, the radius $R_{\odot}=6.96 \times 10^{8} \mathrm{~m}$, and the gravity $g_{\odot}=274.4 \mathrm{~m} / \mathrm{s}^{-2}$ of the Sun, $F r_{\odot}=1.84 \times 10^{-5}$. The difference between its equatorial and the polar radii is about $10 \mathrm{~km}$, so $f l=1.44 \times 10^{-5}$. Moreover, in this problem the negligible centrifugal force does not introduce any component in a direction different from those already given by the projections of $\boldsymbol{g}$, so it cannot produce any new dynamics.

Stress-free, impenetrable $\left(v_{r}=0\right)$, and perfect thermally conducting boundary conditions in terms of the potentials

$$
\Phi=\partial_{r r}^{2} \Phi=\partial_{r}(\Psi / r)=\Theta=0 \quad \text { at } \quad r_{i}=\eta /(1-\eta) \quad \text { and } \quad r_{o}=1 /(1-\eta)
$$

close the formulation of the problem. However, they require enforcing the conservation of the angular momentum in the domain, $\boldsymbol{L}$, during the time integration.

Although this study does not consider the deformation of the surface of the sphere, there are numerous studies ([18-22], among others) devoted to find complete sets of inertial modes (oscillations and waves generated by the Coriolis force) in ellipsoidal fluid volumes, motivated by understanding the liquid core dynamics of deformed planets, and moons. Maffei et al. [21] found complete bases for the inertial waves assuming a quasi-geostrophic motion in a spheroid of stress-free and impenetrable boundaries, and compared the $z$-independent results with those of the three-dimensional problem. Chan et al. [23] investigated the motion of a homogeneous fluid confined in the core of a latitudinally librating planet with finite polar 
excentricity. Inviscid analytical solutions were derived and three-dimensional numerical simulations for viscous fluids at low $E$ were carried out.

The above system is $\mathcal{S O}(2) \times \mathcal{Z}_{2}$-equivariant, $\mathcal{S O}(2)$ generated by azimuthal rotations of an arbitrary angle $\varphi_{0}$, and $\mathcal{Z}_{2}$ by reflections with respect to the equatorial plane, i.e., the actions

$$
R_{\varphi_{0}}: \quad(\Psi, \Phi, \Theta)(t, r, \theta, \varphi) \rightarrow(\Psi, \Phi, \Theta)\left(t, r, \theta, \varphi+\varphi_{0}\right)
$$

and

$$
\zeta_{\theta}: \quad(\Psi, \Phi, \Theta)(t, r, \theta, \varphi) \rightarrow(-\Psi, \Phi, \Theta)(t, r, \pi-\theta, \varphi)
$$

leave the system invariant.

To discretize the system, the functions $X=(\Psi, \Phi, \Theta)$ are expanded in spherical harmonic series up to degree and order $L$ (triangular truncation), namely

$$
X(t, r, \theta, \varphi)=\sum_{l=0}^{L} \sum_{m=-l}^{l} X_{l}^{m}(r, t) Y_{l}^{m}(\theta, \varphi),
$$

with $\Psi_{l}^{-m}=\overline{\Psi_{l}^{m}}, \Phi_{l}^{-m}=\overline{\Phi_{l}^{m}}, \Theta_{l}^{-m}=\overline{\Theta_{l}^{m}}, \Psi_{0}^{0}=\Phi_{0}^{0}=0$ to uniquely determine the two scalar potentials, and $Y_{l}^{m}(\theta, \varphi)=P_{l}^{m}(\cos \theta) e^{i m \varphi}, P_{l}^{m}$ being the normalized associated Legendre functions of degree $l$ and order $m$ with the norms

$$
\sqrt{\frac{2 l+1}{2} \frac{(l-m) !}{(l+m) !}} \quad \text { with } \quad l \geq 0, \quad-l \leq m \leq l .
$$

A collocation method on a mesh of $N_{r}+1$ Gauss-Lobatto points is used in the radial direction. The equations are written in the $N_{r}-1$ inner points by constructing the linear operators fulfilling the boundary conditions. The matrix-vector products to evaluate the radial operators and the Legendre transforms are computed with the optimized GotoBLAS library [24]. More details are given in Ref. [17].

There are different ways of implementing the conservation of $\boldsymbol{L}$. By using an angular discretization in spherical harmonics, the conservation of the components of the angular momentum only depends on the toroidal part of the velocity field, specifically on $\Psi_{1}^{0}(r)$, $\Re\left(\Psi_{1}^{1}(r)\right)$ and $\Im\left(\Psi_{1}^{1}(r)\right)[25,26]$. By initially taking $\boldsymbol{L}=\mathbf{0}$, these radial functions can be treated as $\Psi_{1}^{0}(r)$ in the Appendix of [17], i.e., by modifying in Eq. (2) the parts of the vector field corresponding to these variables. Finally, the system is integrated with the fully implicit BDF method implemented in the subroutine DLSODPK from the ODEPACK package [27], with variable time step and order, and tolerances as low as $10^{-12}$. 


\section{A. Output data definitions}

Aside the analysis of the time series, the dynamics of the three-dimensional flows bifurcating from the axisymmetric periodic branch of solutions are analyzed by monitoring global data obtained by averaging some physical properties of the flows over the whole volume of the sphere, $V$. The volume average of the kinetic energy density, $k=1 / 2(\boldsymbol{v} \cdot \boldsymbol{v})$, is

$$
K(t)=\frac{1}{2 V} \int_{V} k(t, r, \theta, \varphi) d V=\frac{3 \sqrt{2}}{4\left(r_{o}^{3}-r_{i}^{3}\right)} \int_{r_{i}}^{r_{o}} r^{2}\left[v^{2}\right]_{0}^{0}(r, t) d r
$$

where $v^{2}=\boldsymbol{v} \cdot \boldsymbol{v}$, and $\left[v^{2}\right]_{0}^{0}$ means the spherical harmonic coefficient of order and degree zero of $v^{2}$. The same expression can be used for computing the corresponding toroidal, $K_{T}(t)$, poloidal, $K_{P}(t)$, zonal (axisymmetric), $K_{z}(t)$, and nonzonal (non-axisymmetric), $K_{n z}(t)$, parts by replacing $v$ by the corresponding component. In addition, the zonal flow, defined as

$$
\left\langle v_{\varphi}\right\rangle=\frac{1}{2 \pi} \int_{0}^{2 \pi} v_{\varphi} d \varphi
$$

is also tracked.

The temporal average of any of the above functions, $\bar{F}$, over a time interval $\Delta t$ is computed

$$
\bar{F}=\frac{1}{\Delta t} \int_{0}^{\Delta t} F(t) d t
$$

The time integral is approximated by the trapezoidal rule during the time integration. From now on the time and space averaged quantities will be called 'mean quantities' for short.

\section{B. Validation of the results}

To check the convergence of the solutions, some frequencies and mean properties of three quasiperiodic flows were computed with different resolutions.

The initial conditions of the simulations of Table I were obtained by interpolating the coarse grids to finer meshes, and starting the averages once the new solutions reached saturation. The resolution $N_{r} \times L=48 \times 64$ was finally used for $\mathrm{Ra}<12000$, and $N_{r} \times L=64 \times 80$ for the highest Ra, although the change was not strictly necessary because the values given by the two finest grids differ about $1.2 \%$ in the worse case. Moreover, it has been checked that the dynamics described below can be reproduced by using $32 \times 54$ points but with a slight shift of the bifurcation points to lower values. 
TABLE I. Comparison of the main frequencies, $f_{1}$ and $f_{2}$, the mean zonal flow, $\overline{\left\langle v_{\varphi}\right\rangle}$, the total, $\bar{K}$, toroidal, $\overline{K_{T}}$, and poloidal, $\overline{K_{P}}$, mean kinetic energies, with different resolutions and values of $\mathrm{Ra}$ for a time interval $\Delta t>0.5$.

\begin{tabular}{|c|c|c|c|c|c|c|c|c|c|}
\hline$N_{r} \times L$ & $N_{\theta}$ & $N_{\varphi}$ & $\mathrm{Ra}$ & $f_{1}$ & $f_{2}$ & $\overline{\left\langle v_{\varphi}\right\rangle}$ & $\bar{K} \times 10^{-4}$ & $\overline{K_{T}} \times 10^{-4}$ & $\overline{K_{P}} \times 10^{-4}$ \\
\hline $32 \times 54$ & 84 & 168 & 7400 & 1422.66 & 366.13 & $4.672 \times 10^{-2}$ & 1.868 & 0.9615 & 0.9068 \\
\hline $48 \times 64$ & 98 & 196 & 7400 & 1422.69 & 365.91 & $7.018 \times 10^{-2}$ & 1.647 & 0.8394 & 0.8071 \\
\hline $64 \times 80$ & 128 & 256 & 7400 & 1422.70 & 365.88 & $7.047 \times 10^{-2}$ & 1.633 & 0.8316 & 0.8017 \\
\hline $32 \times 54$ & 84 & 168 & 9000 & 1440.08 & 312.17 & -5.598 & 9.204 & 5.237 & 3.967 \\
\hline $48 \times 64$ & 98 & 196 & 9000 & 1439.75 & 312.81 & -5.506 & 9.635 & 5.441 & 4.193 \\
\hline $64 \times 80$ & 128 & 256 & 9000 & 1439.66 & 312.70 & -5.444 & 9.668 & 5.455 & 4.213 \\
\hline $48 \times 64$ & 98 & 196 & 12000 & 1451.56 & 322.53 & -12.379 & 5.804 & 3.800 & 2.005 \\
\hline $64 \times 80$ & 128 & 256 & 12000 & 1451.58 & 322.51 & -12.371 & 5.806 & 3.800 & 2.006 \\
\hline
\end{tabular}
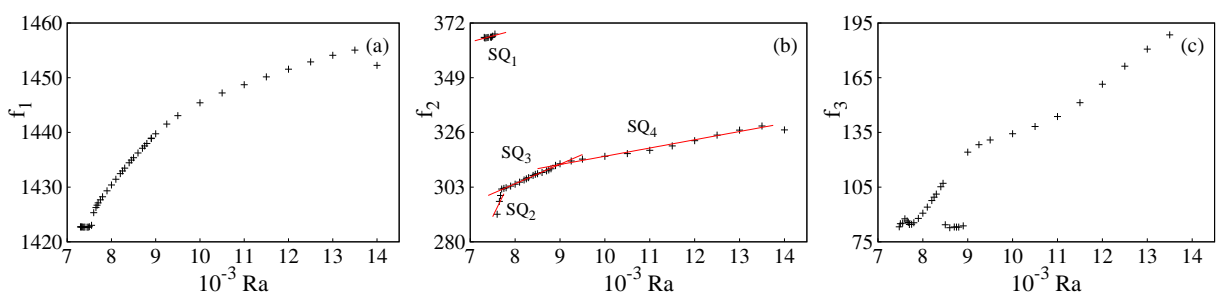

FIG. 2. (a) First (b) second and (c) third main frequencies of the solutions computed.

The solutions of Table I reach better accuracy with $48 \times 64$ points at $\mathrm{Ra}=12000$ than at $\mathrm{Ra}=9000$ because, as will be seen below, the time dependence of the former is simpler.

\section{FREQUENCIES AND MEAN VALUES}

The calculations shown below correspond to a fluid shell with a small gap $\eta=0.01$. In addition $\operatorname{Pr}=10^{-3}, E=10^{-4}$ are taken, to ensure that the first bifurcation from $S_{0}$ is axisymmetric and that the dynamics is dominated by torsional flows.

The integration was started from an interpolated periodic torsional solution obtained for 
the thermal convection of a fluid sphere at $\mathrm{Ra}=8100$ by a continuation method and the parameters and conditions previously mentioned [17]. Therefore, since this bifurcation is supercritical, only solutions above the critical Ra of the onset of convection in this sphere $\left(\mathrm{Ra}_{\mathrm{c}}=7640\right)$ were expected. However, by evolving temporally Eqs. (2)-(4) with boundary conditions (6), the first axisymmetric periodic solution (state $S_{1}$ ) was found at $\operatorname{Ra} \approx 7300$. By increasing Ra from this value, the periodic two-dimensional flow bifurcates quickly to three-dimensional quasiperiodic flows in a second Hopf bifurcation, although the torsional motion is retained. Figures 2(a) and (b) show the two main frequencies of the stable solutions found when $\mathrm{Ra}$ is increased from the lowest solution. Figure 2(a) is that of the main frequency $f_{1}$, associated to the thermal inertial oscillations existent at very low Pr, i.e., it is that of the torsional oscillations. Only the leftmost symbol of Fig. 2(a) corresponds to an axisymmetric solution, but it is impossible to ascertain just by temporal integration whether it is stable or not. The next bifurcation is so close that the transients are too long to conclude something in any sense. When the invariance by rotation is broken the solution starts to drift in the azimuthal direction. The frequency $f_{2}$ of Fig. 2(b) is related to the drift. It is almost four times lower than $f_{1}$, therefore this azimuthal drift is much slower than that of the thermal inertial waves found as primary bifurcation from $S_{0}$ outside the region found in [15].

The change of slope of the first two curves, mainly that of Fig. 2(b), reflects that four sequences of solutions have been found. They are marked on this figure with red straight lines, and also with labels $\mathrm{SQ}_{\mathrm{i}}$, with $i=1, \ldots, 4$. Solutions belonging to $\mathrm{SQ}_{1}$ were found in the range of $\mathrm{Ra}[7300,7550], \mathrm{SQ}_{2}$ in [7600,7675], $\mathrm{SQ}_{3}$ in [7700, 8900], and $\mathrm{SQ}_{4}$ in [9000, 14000]. The first change drives to a slight increase of $f_{1}$, and a decrease of $f_{2}$, and takes place close to $\mathrm{Ra}_{\mathrm{c}}=7640$, i.e. the value expected for the onset of convection in the full sphere. From $\mathrm{Ra} \approx 7600\left(\mathrm{SQ}_{2}\right)$ both frequencies increase substantially up to a second change. Then from $\mathrm{Ra} \approx 7700\left(\mathrm{SQ}_{3}\right)$ there is a decrease of the slope of the curve of frequencies up to a third change that introduces a new slight decrease. From $\mathrm{Ra} \approx 9000\left(\mathrm{SQ}_{4}\right)$ the slope of the curves of frequencies diminish until torus chaos (temporal chaos confined close to the quasiperiodic solution that loses the stability) is reached (last point). However, Fig. 2(c), which shows the main frequency $f_{3}$ of the solutions, suggests that $\mathrm{SQ}_{3}$ includes two different branches. In this interval the frequency $f_{3}$ increases rapidly from $\mathrm{Ra}=7700$, and suddenly recovers its initial value, $f_{3} \approx 84$, around $\mathrm{Ra}=8500$. In any case, these solutions will be analyzed 

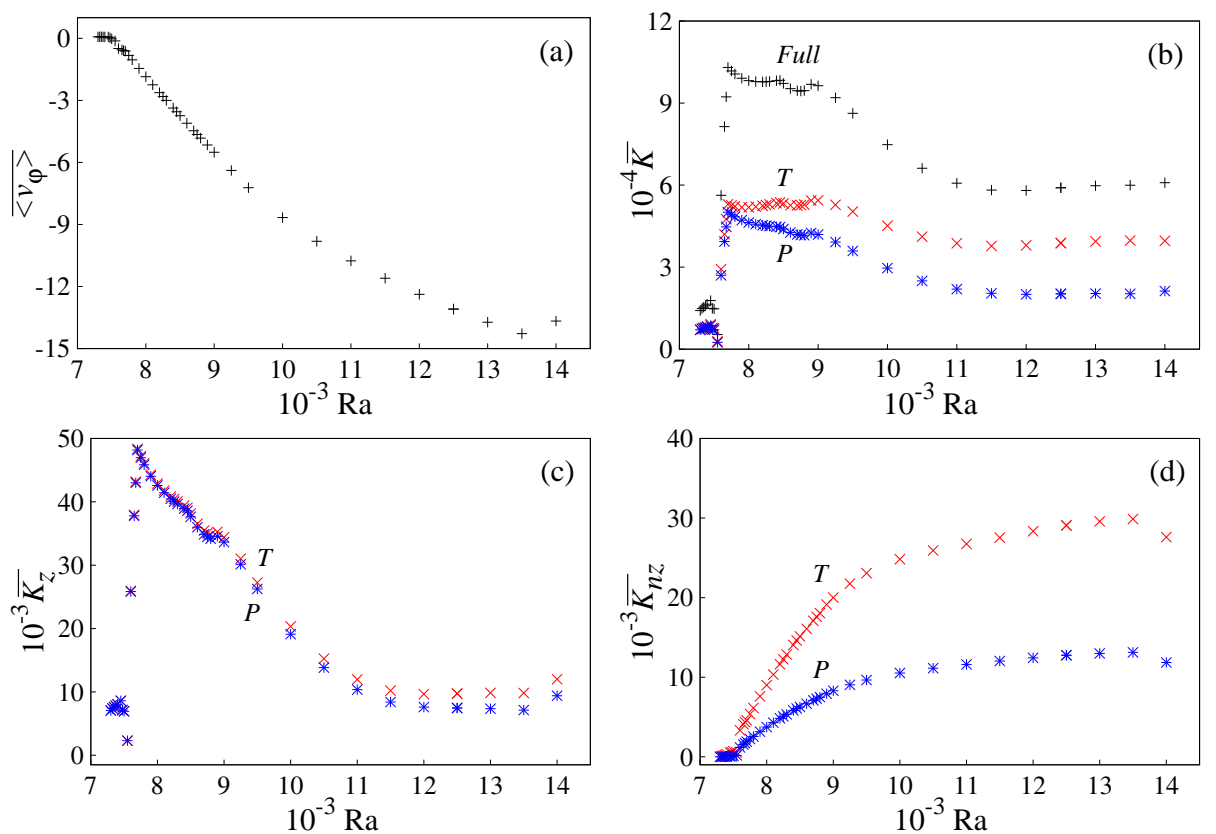

FIG. 3. Mean values of: (a) the zonal flow, $\overline{\left\langle v_{\varphi}\right\rangle}$, (b) the full kinetic energy (black pluses), $\bar{K}$, its toroidal (red crosses), $\overline{K_{T}}$, and poloidal (blue asterisks), $\overline{K_{P}}$, components, of the sequences of stable solutions. Below in (c-d) zonal, $\overline{K_{z}}$, and nonzonal, $\overline{K_{n z}}$, contributions to $\overline{K_{T}}$ and $\overline{K_{P}}$.

together.

No multiplicity of stable solutions by increasing and decreasing the parameter at the jumps between SQ was detected, i.e. hysteresis was not found. However, it is very common to find this phenomena in thermal convection. For instance, the origin of bistability of axisymmetric flows confined between differentially heated spheres at large Pr was studied in [28]. They found that bistability depends on the relative strength of heating and differential rotation, and that the nature of the transition between bistable states depends strongly on Pr.

The same $\mathrm{SQ}_{\mathrm{i}}, \mathrm{i}=1, \cdots 4$, can be seen by depicting the mean zonal flow, $\overline{\left\langle v_{\varphi}\right\rangle}$, (Fig. 3(a)), and the mean kinetic energy, $\bar{K}$, together with its mean toroidal, $\overline{K_{T}}$, and mean poloidal, $\overline{K_{P}}$, components (Figs. 3(b)-(d) respectively). 
TABLE II. Mean values of the zonal flow at selected points $(r, \theta)$ for a solution in each of the four sequences shown in Fig. 3. Columns from left to right correspond to $\mathrm{Ra}=7375,7650,8450,12000$.

\begin{tabular}{l|ccc|ccc|ccc|ccc}
\hline \hline$\theta$ & 0.26 & 0.76 & 1.01 & 0.26 & 0.76 & 1.01 & 0.26 & 0.76 & 1.01 & 0.26 & 0.76 & 1.01 \\
\hline$\pi / 6$ & 0.659 & -0.313 & -2.732 & 0.498 & -7.742 & -19.03 & -7.336 & -26.29 & -37.42 & -31.46 & -73.36 & -75.79 \\
$\pi / 3$ & 1.251 & 0.607 & -2.125 & 1.644 & 0.751 & -6.863 & -11.45 & -6.819 & 7.627 & -52.17 & -38.24 & 47.52 \\
$\pi / 2$ & 1.466 & 1.511 & -1.059 & 2.200 & 8.517 & 1.036 & -12.43 & 11.42 & 25.25 & -58.75 & -6.541 & 130.43 \\
$2 \pi / 3$ & 1.254 & 0.631 & -2.084 & -1.698 & 1.209 & -6.060 & -11.45 & -6.811 & 7.643 & -52.15 & -38.07 & 47.83 \\
$5 \pi / 6$ & 0.662 & -0.294 & -2.691 & 0.547 & -7.316 & -18.28 & -7.335 & -26.28 & -37.41 & -31.44 & -73.21 & -75.51 \\
\hline \hline
\end{tabular}

The mean zonal flow is prograde in $\mathrm{SQ}_{1}$, but very small. After, it becomes retrograde, and its absolute value increases with the increase of Ra. An inspection of the time evolution of $\overline{\left\langle v_{\varphi}\right\rangle}$ and $\left\langle v_{\varphi}\right\rangle$ very near to the points $(r, \theta)=(0.26, \pi / 6),(0.76, \pi / 6),(1.01, \pi / 6),(0.26, \pi / 3)$, $(0.76, \pi / 3),(1.01, \pi / 3),(0.26, \pi / 2),(0.76, \pi / 2),(1.01, \pi / 2)$, and to the symmetric points with respect to the equator in the south hemisphere, shows that, in general, its intensity (either prograde or retrograde) increases from the interior of the sphere to $r_{o}=1.01$. Table II shows typical values at these points for the four sequences found. The mean zonal flow in the north hemisphere and the equator (first three lines) is always very small in $\mathrm{SQ}_{1}$, prograde in the interior and retrograde near and on $r_{o}$. The changes of sign take place between $r=0.26$ and 0.76 at $\theta=\pi / 6$, between $r=0.76$ and 1.01 at $\theta=\pi / 3$, and between $r=0.76$ and 1.01 at $\theta=\pi / 2$. In the second the highest values are retrograde, located at high latitudes (low $\theta$ ) on $r_{o}$. The sign of $\overline{\left\langle v_{\varphi}\right\rangle}$ changes in $\mathrm{SQ}_{2}$ at the low and mid $\theta$, however it remains prograde at the equator, very small in the most internal part and at $r_{o}$. The sequence $\mathrm{SQ}_{3}$ is characterized by a retrograde $\overline{\left\langle v_{\varphi}\right\rangle}$ on $r=r_{o}$ at high latitudes, and prograde at the equator, except in the most internal part. At $\theta=\pi / 3$ there is a change of sign between $r=0.76$ and $r_{o}$. The zonal flow in $\mathrm{SQ}_{4}$ is as in $\mathrm{SQ}_{3}$ except in the equator, with a stronger intensity that increases with Ra. Moreover, $\overline{\left\langle v_{\varphi}\right\rangle}$ in the south hemisphere is almost symmetric to that of the north (two last lines in Table II).

The mean kinetic energy (Full), and its toroidal $(T)$ and poloidal $(P)$ components (Fig. 3(b)) are small at the beginning, growing quickly in $\mathrm{SQ}_{2} . \bar{K}$ becomes almost constant in $\mathrm{SQ}_{3}$, 


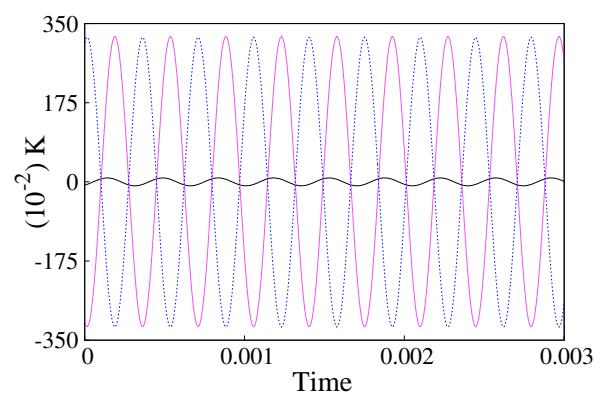

FIG. 4. Oscillation of $K$ (black), $K_{T}$ (magenta solid line) and $K_{P}$ (blue dashed line) around their mean values, which can be seen in Table I. $K$ has been amplified a factor 10 to visualize the oscillation. $\mathrm{Ra}=9000$.

due to a small rise of $\overline{K_{T}}$ and reduction of $\overline{K_{P}}$, with two tiny minima that possibly signal the presence of two different branches of solutions. The sequence $\mathrm{SQ}_{4}$ shows a diminution of the mean kinetic energies, tending finally to an almost constant value.

The contribution of the zonal (axisymmetric), $\overline{K_{z}}$, and nonzonal (non-axisymmetric), $\overline{K_{n z}}$, parts of $\overline{K_{T}}$ and $\overline{K_{P}}$ are shown in Figs. 3(c)-(d). The mean zonal energies are much more important than the nonzonal in $\mathrm{SQ}_{\mathrm{i}}, \mathrm{i}=1,2,3$, despite the opposite slope of the curves from the beginning of $\mathrm{SQ}_{3}$ at $\mathrm{Ra} \approx 7700$. The decrease of $\overline{K_{z}}$ is almost compensated by the increase of $\overline{K_{n z}}$ in $\mathrm{SQ}_{3}$, explaining the almost constant mean energies in the third interval of Fig. 3(b). While the curve of $\overline{K_{n z}}$ is quite smooth that of $\overline{K_{z}}$ shows a cusp at Ra $\approx 8500$, also present in the three curves of Fig. 3(b). In the last segment, both $\overline{K_{z}}$ and $\overline{K_{n z}}$ tend to a constant value while the solutions are quasiperiodic. In any case, the mean zonal energies have a similar value, but the toroidal part of $\overline{K_{n z}}$ is at the end of the interval about three times larger than all the others, giving the main contribution to the full kinetic energy. The increase of $\overline{K_{z}}$ in the last point is compensated by the decrease of $\overline{K_{n z}}$ maintaining the full $\bar{K}$ almost constant when the flow becomes temporally chaotic. This balance of energies differs from that described in Ref. [29] for the thermal Rossby waves and bifurcated flows. They found that the contribution of the zonal $K_{P}$ to $K$ is more than an order of magnitude lower than that of the other three components, while for the torsional flows it is of the same order (see also Table I). 
Although the instantaneous toroidal and poloidal components of the velocity field, $\boldsymbol{v}_{T}$ and $\boldsymbol{v}_{P}$, are not orthogonal, the volume integral of $\boldsymbol{v}_{T} \cdot \boldsymbol{v}_{P}$ vanishes [30], and then $\bar{K}=\overline{K_{T}}+\overline{K_{P}}$ as show Table I and Fig. 3(b). On the other hand there is a periodic interchange of kinetic energy between the toroidal and poloidal parts, inherited from what already happens with the eigenfunction at the onset. Then $K_{T}(t)$ and $K_{P}(t)$ are almost out of phase, and $K(t)$ oscillates with a very low amplitude. Figure 4 illustrates this property for $\mathrm{Ra}=9000$. The volume averaged total energy $K(t)$ has been amplified a factor 10 in order to see clearly the oscillation.

\section{ANALYSIS OF THE SOLUTIONS}

To analyze the bifurcations and transitions that take place along the above curves is difficult because the system has a large size, Pr is very low, and the number of transitions is large in short intervals of Ra, i.e. the solutions are always near a bifurcation or a breakdown.

\section{A. First sequence of solutions $\left(\mathrm{SQ}_{1}\right)$}

As said before, the first axisymmetric periodic solution was found at $\mathrm{Ra}=7300$. The frequency of the solution is $f_{1}=1422.7$, which matches the period, $T_{1}=7.029 \times 10^{-4}$, of the inertial oscillations found in the axisymmetric full sphere problem with the same parameters [17]. For this type of solutions it is known that the torsional velocity field gives rise to a latitudinal propagation of $k$ on the outer surface of the sphere, and to an oscillation of the hot core in the direction of the axis of rotation when Ra is large enough. The periodic oscillations break the equatorial anti-symmetry of the eigenfunction, although near the onset of convection the antisymmetric component is much more important than the symmetric [17]. As said in the introduction, they keep the invariance by rotations, $R_{\varphi_{0}}$ and the spatio-temporal symmetry, then $S_{1}$ fulfills

$$
\begin{aligned}
\left(v_{r}, v_{\theta}, v_{\varphi}\right)(t, r, \theta, \varphi) & =\left(v_{r}, v_{\theta}, v_{\varphi}\right)\left(t+T / 2, r, \pi-\theta, \varphi+\varphi_{0}\right), \\
\mathrm{T}(t, r, \theta, \varphi) & =\mathrm{T}\left(t+T / 2, r, \pi-\theta, \varphi+\varphi_{0},\right)
\end{aligned}
$$

i.e., they are symmetric cycles, which keep the axisymmetry.

The contour plots of the upper row of Fig. 5 correspond to the $S_{1}$ found at $\mathrm{Ra}=7300$. The first three (a)-(c) are snapshots of $\Theta(t)$ and (d)-(f) of $k$ together with the velocity field 

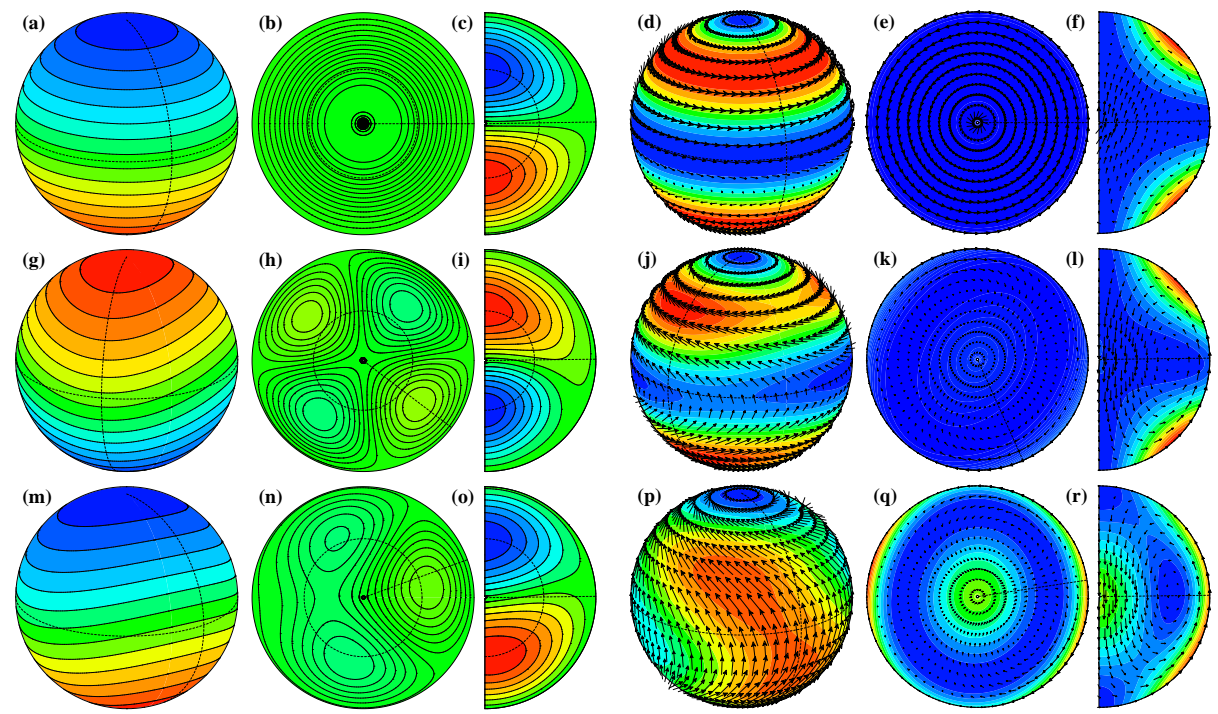

FIG. 5. Contour plots of: (a)-(c), (g)-(i) and (m)-(o) the perturbation of the temperature, $\Theta$, and (d)-(f), (j)-(l) and (p)-(r) the kinetic energy density, $k$, together with the velocity field (arrows), on a spherical surface, the equator and a meridional section, respectively. The first row (a)-(c) and (d)-(f) for $\mathrm{Ra}=7300$, the second (g)-(i) (Multimedia view) and (j)-(l) (Multimedia view) for $\mathrm{Ra}=7375$, and the third (m)-(o) (Multimedia view) and (p)-(r) (Multimedia view) for Ra $=7475$. The place where each projection is taken is marked with dotted lines in the other two.

(arrows). The place where the projections are taken is shown on the own figures. The spherical surfaces (a) and (d) are taken cutting the maximum of the solution, (b) and (e) are equatorial and (c) and (f) meridional sections. The color is global in each group of three projections, but the arrows of the velocity field are scaled for each projection. This criteria will be maintained along the paper. The two local maxima of $k$, one in each hemisphere, are due to the larger weight of the antisymmetric component of the velocity field.

The secondary Hopf bifurcation from $S_{0}$ breaks the axisymmetry of $S_{1}$ (symmetry $R_{\varphi_{0}}$ ), but the emerging invariant two-dimensional tori, $\mathbb{T}^{2}$, retain the spatio-temporal symmetry as sets, and the instantaneous invariance under arbitrary azimuthal rotations of $\varphi_{0}=\pi$ $\left(R_{\pi}\right)$. It makes the fluid to start to drift in the azimuthal direction (state $S_{2}$ ). An analogous 


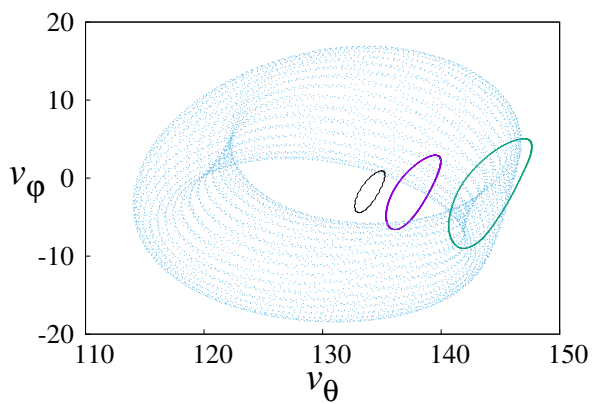

FIG. 6. Projections of the Poincaré sections showing the value of $v_{\theta}(0.26, \pi / 6,0)$ versus that of $v_{\varphi}(1.01, \pi / 6,0)$ on the hyperplane $v_{\varphi}(0.76, \pi / 6,0)=0$. The values of Ra from the smaller to the larger sections are 7325 (black), 7375 (purple), 7450 (teal), and 7475 (blue).

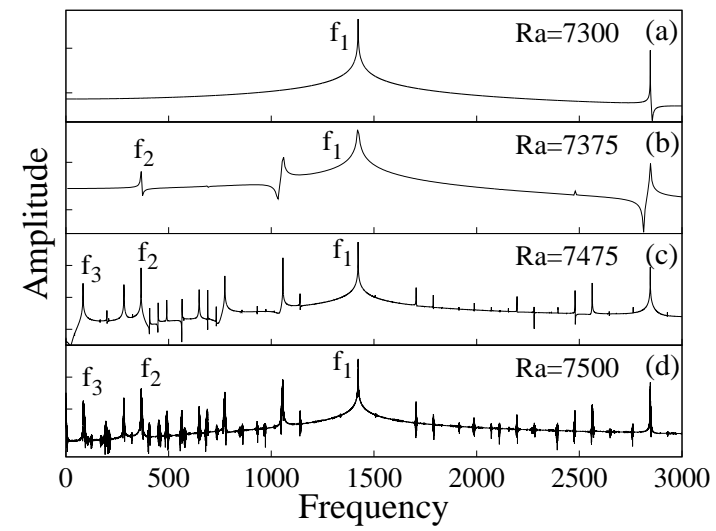

FIG. 7. Spectra of $v_{\varphi}(1.01, \pi / 6,0)$ for (a) Ra $=7300$, (b) 7375 , (c) 7475 , and (d) 7500 .

drift appears when the axisymmetry of a steady flow is broken in a Hopf bifurcation (see for instance [31]). The difference is that in this case the transition leads to a quasiperiodic flow (trajectory on a $\mathbb{T}^{2}$ ) instead of a periodic orbit. The bifurcation takes place around $\mathrm{Ra}=7312$. Since it keeps the invariance $R_{\pi}$, only the spherical harmonics of order multiple of $m=2$ contribute to the expansion (9) of the new solution. The inverse of the frequency $f_{2}$ 


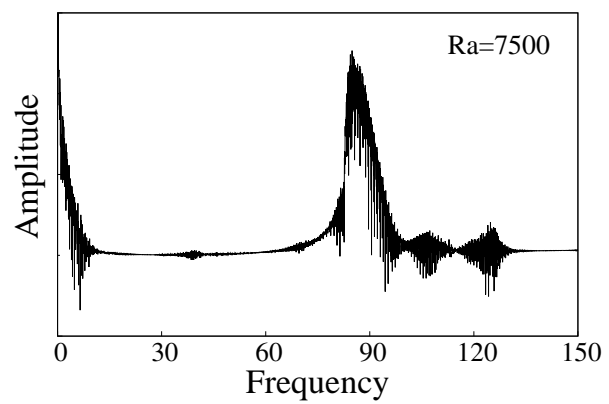

FIG. 8. Detail of Fig. $7(\mathrm{~d})$ around the peak $f_{3}=84.95$.

approximates the time taken by $k$ to drift an angle $\pi$. The state $S_{2}$ satisfies instantaneously

$$
\begin{aligned}
\left(v_{r}, v_{\theta}, v_{\varphi}\right)(t, r, \theta, \varphi) & =\left(v_{r}, v_{\theta}, v_{\varphi}\right)(t, r, \theta, \varphi+\pi), \\
\mathrm{T}(t, r, \theta, \varphi) & =\mathrm{T}(t, r, \theta, \varphi+\pi) .
\end{aligned}
$$

and as a set $\zeta_{\theta}\left(\mathbb{T}^{2}\right)=\mathbb{T}^{2}$. This means that if $X$ is a point on the torus, then so is $\zeta_{\theta}(X)$.

The second row of Fig. 5 shows the same snapshots for $\mathrm{Ra}=7375$ as for $\mathrm{Ra}=7300$. The two-fold symmetry and the prograde drift of the solutions can be clearly observed in the animations of the equatorial projection of $\Theta(t)$ in Fig. 5(g)-(i) (Multimedia view), and in the projection of $k$ on the outer surface of Fig. 5(j)-(l) (Multimedia view). The wave of $k$ traveling latitudinally from the equator to mid-latitudes is better seen in the meridional section. The maxima of $k$, one in each hemisphere, travel from mid latitudes to the equator, joining and splitting there at each torsional oscillation. Their double direction of propagation gives rise to a meandering path on the outer spherical surface. This solution has $f_{1}=1422.7$ and $f_{2}=365.88$.

The state $S_{2}$ loses stability through a third Hopf bifurcation that breaks the invariance $R_{\pi}$, and gives rise to a branch of quasiperiodic solutions with three fundamental frequencies, then the trajectories are on invariant three-tori, $\mathbb{T}^{3}$, (state $S_{3}$ ). The transition takes place around $\mathrm{Ra}=7450$. The third row of Fig. 5 shows the contour plots of the new solution at $\mathrm{Ra}=7475$, for which $f_{1}=1422.7, f_{2}=366.02$ and $f_{3}=83.25$. Once again the equatorial section of $\Theta(t)$ and the projection of $k$ on the outer surface reflects the symmetry breaking. Despite this fact, hints of the previous symmetry can still be seen following the waves on 


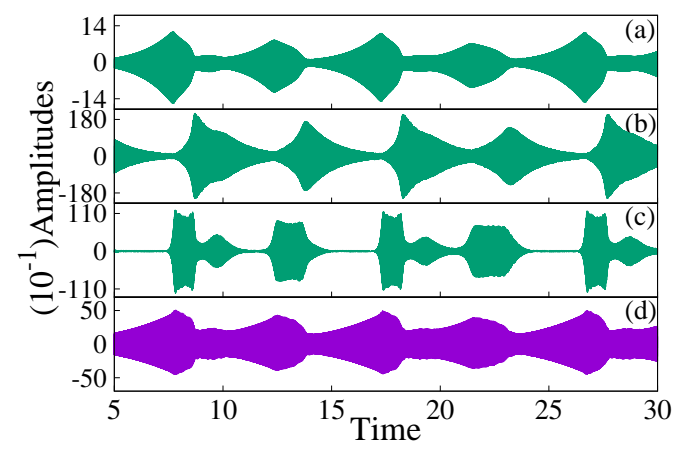

FIG. 9. Time evolution, close to a heteroclinic chain, of the modes (a) $\Theta_{0}^{0}$, (b) $\Theta_{1}^{1}$ and (c) $\Theta_{2}^{2}$, at the radial point $r=0.76$, and (d) azimuthal component $v_{\varphi}(1.01, \pi / 6,0) . \mathrm{Ra}=7500$.

the animations of Fig. 5(m)-(o) (Multimedia view) and on Fig. 5(p)-(r) (Multimedia view). Near the bifurcation point the splitting of $k$, traveling simultaneously up and down between the equator and both poles, remains because the growing modes of order $m=1$ are still small.

The projection of the Poincaré sections (PS from now on) in Fig. 6 and the Fourier spectra of the solutions in Fig. 7(a)-(c) confirm this sequence of bifurcations. The first three sections are closed curves indicating that they correspond to states $S_{2}$. The state $S_{3}$ is represented by a wide band of points. The new frequencies appearing at each bifurcation are labeled on Fig. $7(\mathrm{a})-(\mathrm{c})$, which contains the spectra of $v_{\varphi}(1.01, \pi / 6,0)$.

The last solutions lose stability soon. By increasing Ra, instead of getting a chaotic attractor with a continuum spectrum of frequencies, solutions whose main peaks coincide with those of Fig. 7(a)-(c) are found (see Fig. 7(d)). However, the quasiperiodicity is broken by a very low new frequency that introduces noise around the peaks found at lower Ra. For instance, at $\mathrm{Ra}=7500$ this frequency is 0.21 . Figure 8 shows a detail of this noise around the peak $f_{3} \approx 85$. The temporal evolutions of Fig. 9 of one of the stable solutions found in the range $7475<\mathrm{Ra}<7600$ reveals that the solution is close to a heteroclinic chain jumping among low and high amplitude states. Figure 9(a)-(c) correspond to the modes $\Theta_{0}^{0}, \Theta_{1}^{1}$ and $\Theta_{2}^{2}$ at $r=0.76$, respectively, and the lower row to $v_{\varphi}(1.01, \pi / 6,0)$. The three-dimensional projection of the PS close to the heteroclinic chains confirm this hypothesis (see Fig. 10). 


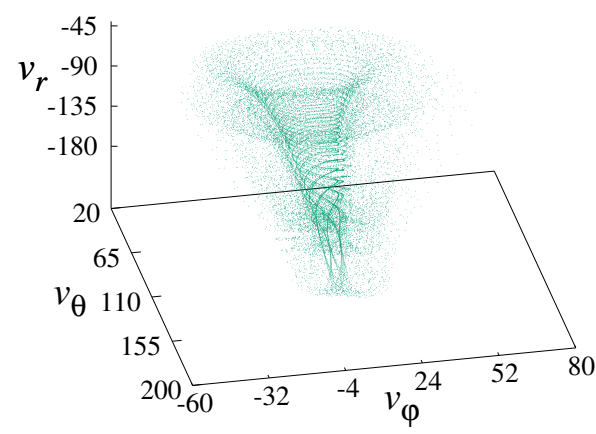

FIG. 10. Three-dimensional projection of the Poincaré section showing the values of $v_{\theta}(0.26, \pi / 6,0)$, $v_{\varphi}(1.01, \pi / 6,0)$, and $v_{r}(0.76, \pi / 6,0)$ on the hyperplane $v_{\varphi}(0.76, \pi / 6,0)=0$ for $\operatorname{Ra}=7500$.

The section shows the three components of the velocity field $v_{\theta}(0.26, \pi / 6,0), v_{\varphi}(1.01, \pi / 6,0)$, and $v_{r}(0.76, \pi / 6,0)$ when the hyperplane $v_{\varphi}(0.76, \pi / 6,0)=0$ is crossed. The trajectories of this type of solutions spiral, approaching two different tori of high amplitude and a periodic orbit that would be at the vertex of the cone-like structure of Fig. 10. In addition, the snapshots show that during the time intervals of the highest amplitude of the $m=2$ modes, the contour plots resemble a $S_{2}$ state, nearly satisfying the $\pi$-invariance by rotations, while during the time intervals of the highest amplitude of the $m=1$ modes, the contours plots resemble a $S_{3}$ state. When all these modes have a low amplitude the orbit approaches a $S_{1}$.

\section{B. Second sequence of solutions $\left(\mathrm{SQ}_{2}\right)$}

As shown in Fig. 2 and Fig. 3 the main frequencies and mean kinetic energies along $\mathrm{SQ}_{1}$ are almost constant. From Ra $>7550$, a second sequence $\mathrm{SQ}_{2}$ characterized by a fast increase of $\overline{\left\langle v_{\varphi}\right\rangle}$ and $\bar{K}$ is found. It is a branch of states $S_{3}$ without spatial symmetries, therefore the modes of order $m=1$ are not null. The contour plots of Fig. 11 show the solution obtained at $\mathrm{Ra}=7650$. The equatorial projection of $\Theta$ and those of $k$ reflect the decrease of the weight of the modes $m=2$ in this sequence, but some hints of their contribution remain, as can be observed in Fig. 11(a)-(c) (Multimedia view) and in Fig. 11(d)-(f) (Multimedia view). Most of the time there is only a maximum of $k$ on the surface of the sphere, and the torsional dynamics and the meandering propagation of $k$ is maintained. However, as 

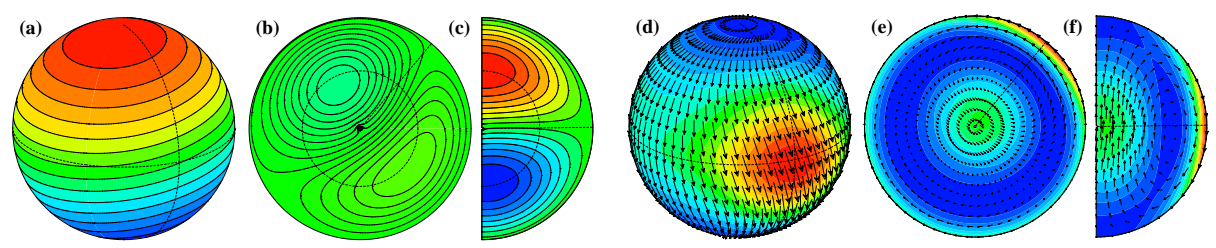

FIG. 11. Same contour plots as in Fig. 5 for $\mathrm{Ra}=7650$. (a)-(c) for $\Theta$ (Multimedia view) and (d)-(f) for $k$ (Multimedia view).

can be seen in the animation of Fig. 11(d)-(f) when the flow is affected by the perturbation introduced by $f_{3}$ there is an enlargement of the spot forming a double maxima at similar latitudes, and recovering the original shape after approximately half a turn. The rotation of the velocity field on the spherical section is quite constant, as happens for $S_{1}$ after the onset.

By increasing Ra it is relatively easy to find resonances that can only be identified by looking at the PS. It is not possible to distinguish these solutions by the spectra because they have essentially the same main peaks, and neither to appreciate differences in the velocity field with the naked eye. For instance, in the two spectra of Fig. 12 one of them belongs to a $S_{3}$, with the trajectories on an invariant $\mathrm{T}^{3}$, and the other to a resonant (or mode-locked) solution (state $R S_{3}$ ). However, the PS of Fig. 13 evidence the dissimilarity between them. The solution at $\mathrm{Ra}=7650$ is resonant since the PS is a closed curve. This means that there is a linear combination of the three frequencies verifying $m_{1}\left(f_{1} / f_{3}\right)+m_{2}\left(f_{2} / f_{3}\right)=m_{3}$, with non-null integers $m_{1}, m_{2}$, and $m_{3}$, and $f_{1} / f_{3}$ and $f_{2} / f_{3}$ not rationals [32, 33]. The inverse of the frequency $f_{2}$ approximates the time taken by the spot of $k$ to drift a full turn, so its propagation is much faster than in $\mathrm{SQ}_{1}$. In contrast the solutions at $\mathrm{Ra}=7600$ and 7675 fill densely a band because the linear combination is only fulfilled with $m_{1}=m_{2}=$ $m_{3}=0$. Apart from computing the spectra via FFTs, their main frequencies were also calculated by the NAFF method (numerical analysis of fundamental frequencies) [34], and some of them by a DMD method (dynamic mode decomposition method) [35], to identify the resonances. Since the accuracy of the frequencies is limited, the resonance conditions can only be established in an approximate way. For instance, for $\mathrm{Ra}=7650$ the best low-order combination $m_{1}\left(f_{1} / f_{3}\right)+m_{2}\left(f_{2} / f_{3}\right)=m_{3}$ with $m_{1}=-1, m_{2}=6$ and $m_{3}=4$ gives an error 
of $11 \%$. However, other resonances were determined with the same criteria, giving much higher precision (see below the case of $\mathrm{Ra}=12000$ ).

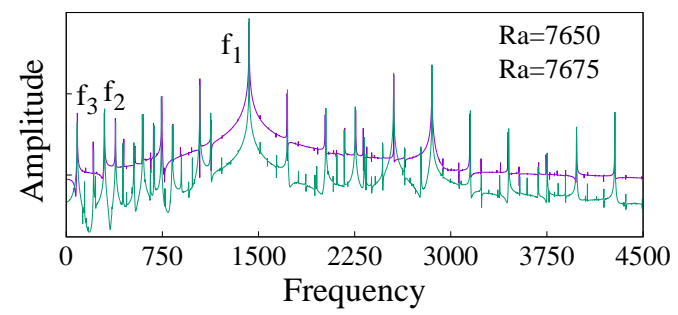

FIG. 12. Spectra of $v_{\varphi}(1.01, \pi / 6,0)$, the upper for $\mathrm{Ra}=7650$ (purple), and the lower for 7675 (teal).

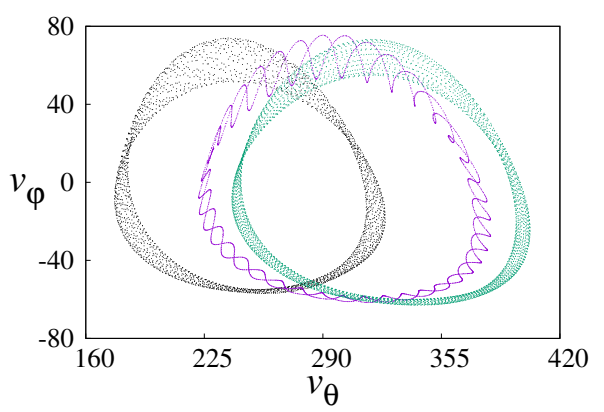

FIG. 13. Idem Fig. 6 for Ra $=7600$ (black), 7650 (purple), and 7675 (teal).

\section{Third sequence of solutions $\left(\mathrm{SQ}_{3}\right)$}

The third sequence, $S Q_{3}$, includes the interval $7700<\mathrm{Ra}<9000$, and it is characterized by a drastic decrease of the modes of order $m=2$. The perturbation of the temperature looks similar to that of Fig. 11(a)-(c), and $k$ is much more localized than before, with a single spot meandering on the surface of the sphere between high mid-latitudes of both hemispheres. 


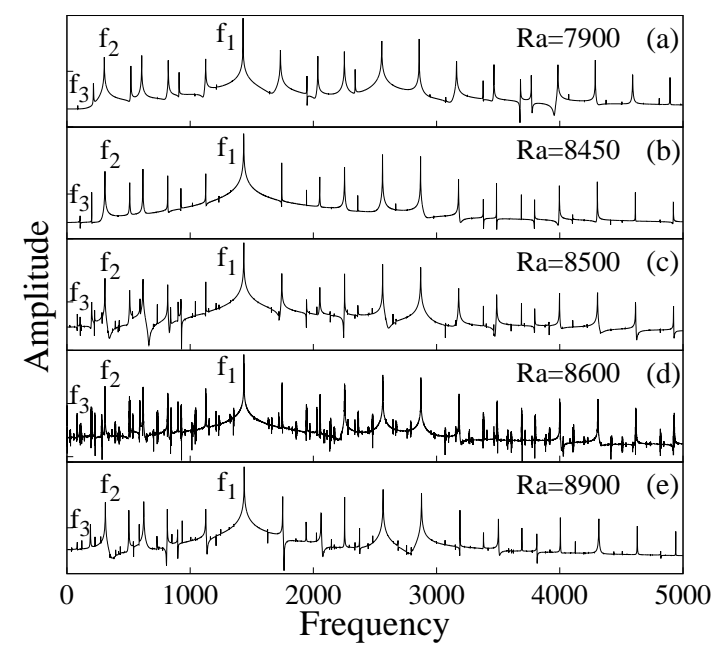

FIG. 14. Spectra of $v_{\varphi}(1.01, \pi / 6,0)$ for (a) Ra $=7900$, (b) 8450, (c) 8500, (d) 8600, and (e) 8900 .

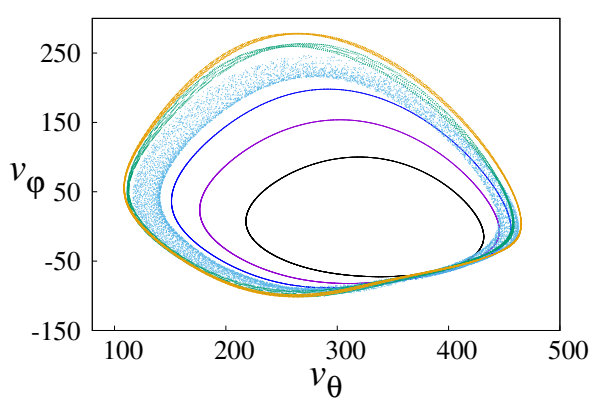

FIG. 15. Idem Fig. 6, the smallest for Ra $=7900$ (black), 8200 (purple), 8450 (dark blue), 8600 (blue), 8800 (teal), and the largest for 8900 (golden).

The Fourier spectra of Fig. 14 and the Poincaré maps of Fig. 15 show the transitions on $\mathrm{SQ}_{3}$. There are three main frequencies in the spectra from the beginning. All of them are of the order of those found in Sec. IV B. The first two display a weak increase, but the third grows much faster with Ra in the interval $7700<\mathrm{Ra}<8450$ (see Fig. 2). The PS are bands of tiny width, which need and enlargement to be seen (shown in Fig. 15 and in the 
details of Figs. 16(a)-(d)). The third frequency $f_{3}$ just introduces a slight perturbation to the two-frequency flows. However, the ratios $f_{1} / f_{3}$ and $f_{2} / f_{3}$ have a strong variation among the solutions due to the fast increase of $f_{3}$. These ratios determine the type of quasiperiodicity on the $\mathbb{T}^{3}[36,37]$. Despite the spectra of Figs. 14(a) and (b) have the same high peaks, at $\mathrm{Ra}=7700, f_{3}=84.5$ and at $\mathrm{Ra}=8450$ it is already 107.1, and the PS look very different, mainly when they are $R S_{3}$ (or near) as those of Figs. 16(c)-(d). These sections were drawn once very long transients are passed, and any of them contains more than 30000 points corresponding to long integrations of $\mathcal{O}(10)$ viscous time units.

When $\mathrm{Ra}>8450$ the mean value of $\bar{K}$ starts to decrease, the solutions recover the third frequency $f_{3} \approx 84$ and in addition new peaks below that of $f_{3}$ appear for the first time in the spectra (see Fig. 14(c)-(d)). The PS become clear wide bands (see the wider blue band of Fig. 15 belonging to $\mathrm{Ra}=8600)$, but their structure changes a lot in the solutions studied. At $\mathrm{Ra}=8500$ the lower frequency is $f_{4}=23.4$, while at higher values a new additional order one frequency appears, which introduces clusters of peaks in the spectrum around the already existent (see Fig. $14(\mathrm{~d})$ ). At Ra $\approx 8750$, which is very close to the second minimum of $\mathrm{SQ}_{3}$ in Fig. 3(b), the spectra change again. The order one frequency disappears for $\mathrm{Ra}>8750$ (two biggest thin bands in Fig. 15). At Ra $=8800$ the solution is close to a $R S_{3}$ (Fig. 16(e)), and it is a $R S_{3}$ at Ra $=8900$ (Fig. 16(f)), near the crossing with $\mathrm{SQ}_{4}$ (see Fig. 3).

\section{Fourth sequence of solutions $\left(\mathrm{SQ}_{4}\right)$}

The sequence $\mathrm{SQ}_{4}$ begins with a spectra of three main frequencies, but the small frequency about 84 never appears in the spectra. The first stable solution was found at $\mathrm{Ra} \approx 9000$ with the frequencies $f_{1}, f_{2}$ of Table $\mathrm{I}$ and $f_{3}=124.19$. The flow does not retain any instantaneous symmetry, and its dynamics is very similar to that of states $\mathrm{S}_{3}$ described in Sec. IV C. Along $\mathrm{SQ}_{4}$ the torsional oscillations and the drift become more intense and faster. Fig. 17(a)-(c)(Multimedia view) illustrates the oscillations of the hottest core of the fluid when Ra increases. The eccentricity of the core (in red) is clear by comparing its contour line with the dotted lines, which mark the place where the spherical projection is taken. Fig. 17(d)-(f)(Multimedia view) evidences that if Ra increases $k$ becomes more and more confined. 

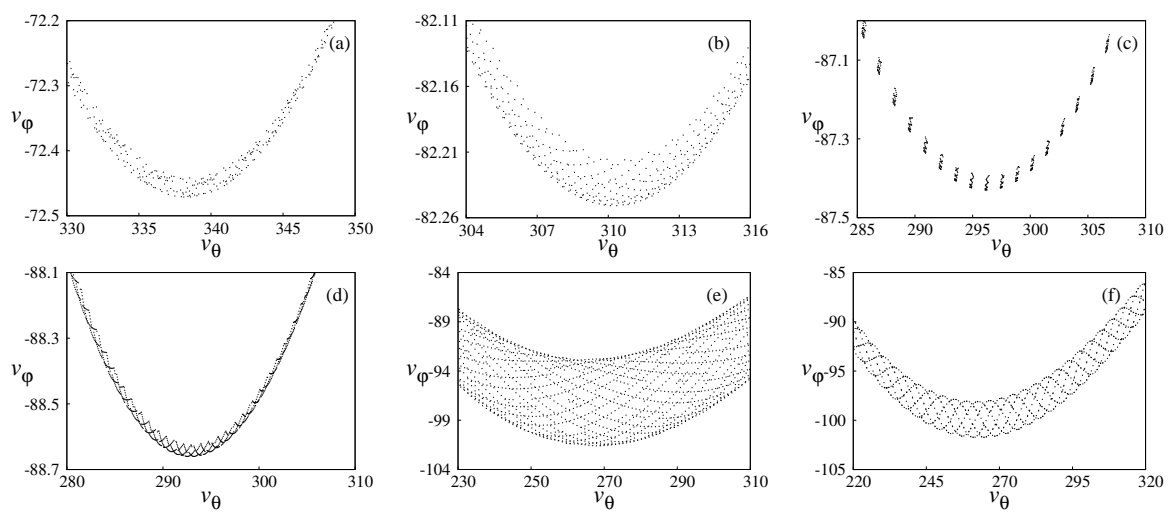

FIG. 16. Details of Fig. 15 showing states: (a) and (b) $S_{3}$ at $\mathrm{Ra}=7900$ and $\mathrm{Ra}=8200$, (c) $R S_{3}$ at $\mathrm{Ra}=8400,(\mathrm{~d})$ and (e) near $R S_{3}$ at $\mathrm{Ra}=8450$ and $\mathrm{Ra}=8800$, and (f) $R S_{3}$ at $\mathrm{Ra}=8900$.
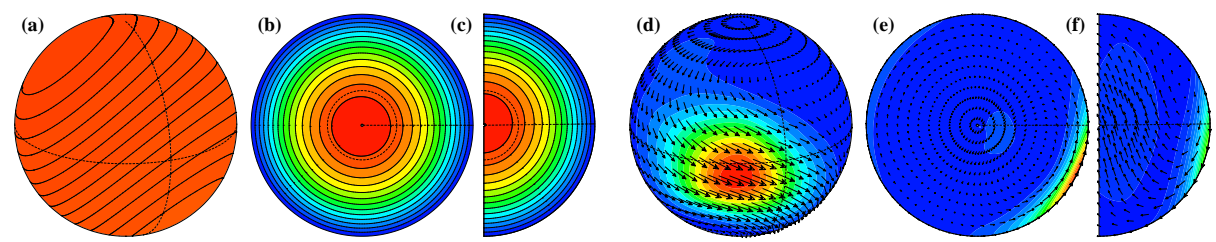

FIG. 17. (a)-(c) Total temperature, $T=T_{c}+\Theta$, (Multimedia view) and (d)-(f) $k$ (Multimedia view) for $\mathrm{Ra}=14000$.

When Ra grows $f_{3}$ starts to grow as well, while the frequency of the next peak of the spectrum starts to decrease (Figs. 18(a)-(c)). Finally both peaks collide at $\mathrm{Ra}=12000$ (Fig. 18(d)). It has been checked that when this happen there is a quasiperiodic frequencylocking with $m_{1}=1, m_{2}=-4$ and $m_{3}=1$. The main frequencies are $f_{1}=1451.56$, $f_{2}=322.53$, already included in Table $\mathrm{I}$, and $f_{3}=161.47$, giving a relative error for the resonance condition below $10^{-3}$. At higher Ra the peaks separate again, they interchange their positions (Fig. 18(e)), and finally at $\mathrm{Ra}=14000$ a noisy spectrum of lowest significant frequency $f=15.76$ is found. In this case the peaks $f_{1}$ and $f_{2}$ can be identified in the spectrum because they almost keep the value of the previous computed solution, but not $f_{3}$ (Fig. 18(f)). 


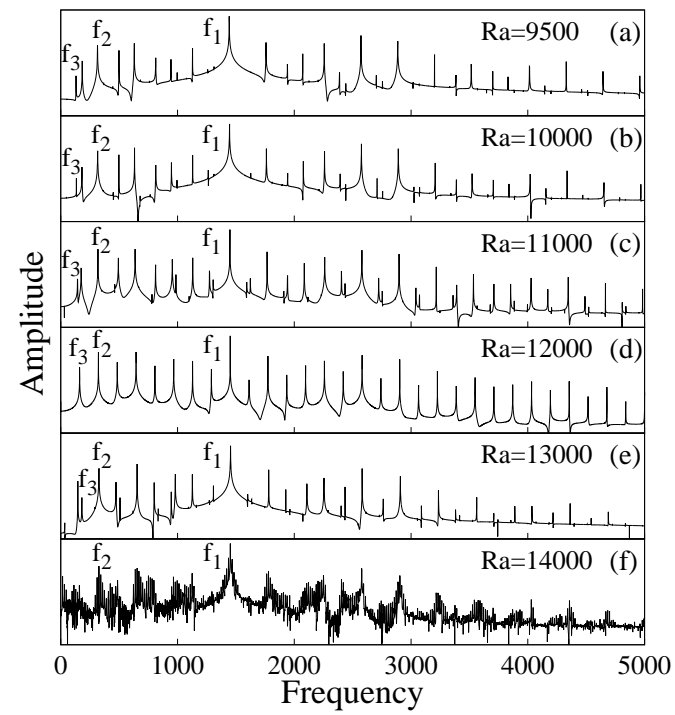

FIG. 18. Spectra of $v_{\varphi}(1.01, \pi / 6,0)$ for (a) Ra $=9500$, (b) 10000, (c) 11000, (d) 12000, (e) 13000, and (f) 14000 .

Figure 19(a) shows the growth of the PS of the solutions on $\mathrm{SQ}_{4}$. In this case the hyperplane $v_{\varphi}(0.76, \pi / 6,0)+v_{\varphi}(1.01, \pi / 6,0)=-142$ was taken because the transversality of the trajectories to that given by $v_{\varphi}(0.76, \pi / 6,0)=0$ is lost. The enlargement of the PS shows that the behavior of the quasiperiodic tori is similar to that found in Sec. IV C, with rotation numbers even lower. Figure 19(b) shows a detail of the section at Ra $=12000$ when the two peaks merge. The frequency-locking gives rise to a closed curve. The cloud of disperse points corresponds to $\mathrm{Ra}=14000$. The appearance of very low frequencies leads to a wide section of disperse points, which is an indication that the flow is temporally chaotic (see Sec. V). Even in this case the torsional dynamics and longitudinal direction of propagation are maintained. 

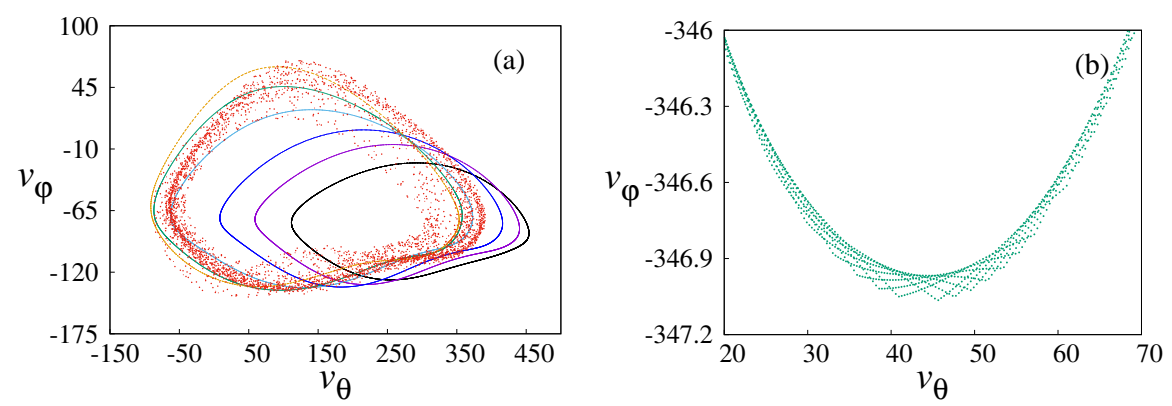

FIG. 19. (a) Projection of the Poincaré section showing $v_{\varphi}(1.01, \pi / 6,0)$ versus $v_{\theta}(0.26, \pi / 6,0)$ on the hyperplane $v_{\varphi}(0.76, \pi / 6,0)+v_{\varphi}(1.01, \pi / 6,0)=-142$ for $\mathrm{Ra}=9000$ (smallest black), 9500 (purple), 10000 (dark blue), 11000 (blue), 12000 (teal), 13500 (golden), and 14000 (brown disperse points). (b) Detail of (a) for $\mathrm{Ra}=12000$.

\section{BREAKDOWN OF THE THREE-DIMENSIONAL TORI AND CHAOTIC AT- TRACTORS}

In order to clarify the complex behavior described, on the one hand we have computed the distance from the points of a trajectory on the attractor to those of another trajectory started by perturbing the initial condition of the former. Past a fast initial decrease of the distance due to the attracting nature of the invariant solutions, it must increase linearly if the solution is quasiperiodic, and it must increase exponentially for chaotic solutions. On the other hand the results have been compared with those found in Refs. [32, 36-38], which study the dynamics in three-tori of low-dimensional systems, and the transition from highdimensional tori to torus chaos or more complex chaotic attractors. These models allow to explore deeply the range of parameters, and to compute the Lyapunov exponents to determine when and how the dynamics becomes chaotic.

The distance between trajectories was computed for solutions with very different Poincaré maps. Those for $\mathrm{Ra}=8400,8600,12000$ and 14000 were selected. As expected, the best fit of the distance for solutions at $\mathrm{Ra}=8400$ and 12000 is linear, but the same happens with the solution found at $\mathrm{Ra}=8600$ whose spectrum seemed to belong to temporal chaotic solutions. Figure. 20(a) shows a temporal evolution of the distance for this Ra, including the 
linear fit (red straight line). It is probably a four-dimensional tori, despite N-dimensional tori with $\mathrm{N} \geq 3$ become exponentially rare when $\mathrm{N}$ is increased [37]. To confirm this hypothesis, linear combinations of the frequencies of the spectrum taking $3\left(f_{1}=1436.24\right.$, $\left.f_{2}=309.15, f_{3}=82.75\right)$ or $4\left(f_{1}=1436.24, f_{2}=309.15, f_{3}=82.75, f_{4}=0.832\right)$ main frequencies were calculated, and the resulting errors were compared. The combination with 4 frequencies, with the same restriction in the order of the combination as those of 3 , gave errors at least two orders of magnitude lower for any of the frequencies, suggesting the presence of high-dimensional tori in $\mathrm{SQ}_{3}$. The existence of high-dimensional torus in a wide range of parameters is in agreement with the results of Ref. [32]. On the contrary, Fig. 20(b)
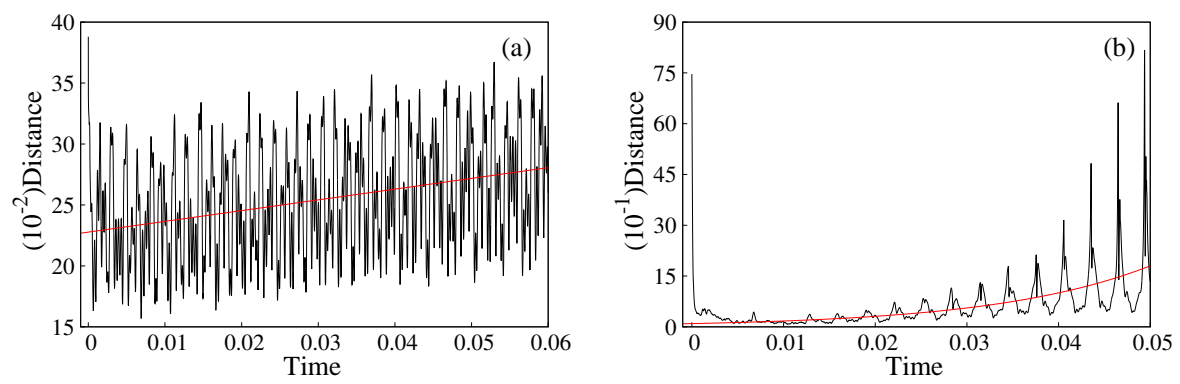

FIG. 20. Distance between two trajectories (the second started by perturbing the initial condition of the former), and in red the corresponding fit, linear for (a) Ra $=8600$, and exponential for (b) $\mathrm{Ra}=14000$.

shows that at $\mathrm{Ra}=14000$ the distance grows exponentially confirming that the solution is chaotic. The red curve is the fit to the temporal increase of the distance. The different structure between both attractors was also supported by the fact that other projections of other variables on the same hyperplane maintain the toroidal form at $\mathrm{Ra}=8600$, while at 14000 disperse clouds of points filling the volume around the torus are found. Yamagishi and Kaneko [37] suggest the fractalization of the three-torus at the transition to chaos. No hint of this behavior was found here.

The comparison of ours results, mainly the structure of the PS, with those plotted in Ref. [36], which study the dynamics on three-dimensional tori and in quasiperiodic Arnold tongues of a four-dimensional coupled delayed logistic map, shows that the dynamics found here from $\mathrm{Ra} \approx 7600$ is what must be expected in the vicinity of codimension-two quasiperi- 
odic Hopf and saddle-node bifurcations [38]. The two-dimensional tori are located inside the quasiperiodic Arnold tongues, just as periodic solutions are located inside the conventional ones. In order to have clearer sections for the solutions like those of Figs. 16(c) and (d), which are very close to a quasiperiodic Arnold tongue, they should be computed several times until reaching the parameters corresponding to the tongues. Simulating high-dimensional systems with long relaxation transients due to small and very different diffusive scales is too expensive, and out of the scope of this study. In any case, we have checked, by recomputing some of the results of Ref. [36] and finding the frequency spectra, that they resemble those of our solutions with similar PS.

\section{CLOSING REMARKS}

Although the purely $S_{1}$ flows are either stable in a very short range of Ra or they could even be unstable from the beginning due to a subcritical Hopf bifurcation if the domain is a spherical shell, this study shows that the bifurcated flows maintain the torsional motion when they travel azimuthally due to the breaking of the axisymmetry, and even when they become temporally chaotic. The two main frequencies, $f_{1}$, linked to the inertial oscillations, and $f_{2}$, linked to the azimuthal drift of the wave, are present in the spectra of all the flows.

This study does not clarify if the introduction of a small inner core leads to a subcritical bifurcation from $S_{0}$ or simply advances the onset of convection with respect to that of the full sphere. Both scenarios are compatible with $\mathrm{SQ}_{1}$. The origin of other sequences remains unclear since they seem disconnected from the basic branch. In any case, the stability problem of the initial axisymmetric solutions should be solved to clarify the bifurcation diagram.

Table II shows that the torsional flow generates only a weak retrograde zonal flow on the surface of the sphere at high latitudes at the onset of the convection. When $\mathrm{Ra}>7700$ a strong prograde jet is located at the equator. In addition two retrograde jets of high intensity coexist at high latitudes, like the zonal flows found by Christensen [39], among others, for $\operatorname{Pr}=\mathcal{O}(1)$. Due to this mixed motion in latitude and longitude, the maximum of the kinetic energy travels meandering on the surface of the sphere.

Quasiperiodic tori, heteroclinic chains, alternating quasiperiodic solutions of three, two (frequency-locked), or even four main frequencies, and chaotic attractors were found along 
the sequences of solutions obtained by increasing $\mathrm{Ra}$ and depending on the ratios of frequencies. However, new frequencies, from the third, only introduce long time perturbations to the torsional-azimuthal wave motion, highlighting its robustness. It is known that quasiperiodic and chaotic axisymmetric torsional motions generate and sustain magnetic fields [16], therefore it would be interesting to find out if the three-dimensional torsional flows traveling azimuthally are also able to generate the same type of magnetic fields, and what is their structure.

The comparison of the results found in this work with those of Ref. [14] show that low Pr fluids have very different three-dimensional dynamics when the parameters are changed. In Ref. [14] the azimuthal waves seem to bifurcate directly from the conduction state and travel in the retrograde direction, while in the present case, at least at the beginning, they come from a Hopf bifurcation on the branch of $S_{1}$ states, and in any case they travel in the prograde direction. The azimuthal waves subject to torsional dynamics are completely different to the very well known Taylor columns and bifurcated flows, so they can contribute to understand other phenomena like the dynamics of the torsional oscillations in the Sun, whose origin is not completely explained or the formation of localized spots of high kinetic energy on the atmospheres of the planets.

The transition from high-dimensional tori to chaos is not fully understood even in low dimensional systems. Examples appearing in particular physical problems, like those shown here, can help to establish what are the generic mechanisms of these transitions. In addition, the existence of flows that evolve to more complex temporal dynamics, when the parameters are moved, through consecutive Hopf bifurcations or via the appearance of heteroclinic chains visiting different invariant objects (equilibria, relative equilibria, periodic orbits, tori, etc.), described by some authors as coherent structures, reinforces the idea that this could be a path to the generation of some kinds of turbulence in fluids [40, 41].

\section{ACKNOWLEDGMENTS}

This research has been supported by the Spanish Ministry of Economy and Competitiveness and the European Regional Development Fund, under Grants MINECO/FEDER FIS2016-76525-P. We are indebted to Prof. Àngel Jorba from Universitat de Barcelona for his help in interpreting the dynamics of the high-dimensional tori. 


\section{DATA AVAILABILITY STATEMENT}

The data that support the findings of this study are available from the corresponding author upon reasonable request.

[1] C. Guervilly and P. Cardin, "Subcritical convection of liquid metals in a rotating sphere using a quasi-geostrophic model," J. Fluid Mech. 808, 61-89 (2016).

[2] E. J. Kaplan, N. Schaeffer, J. Vidal, and P. Cardin, "Subcritical Thermal Convection of Liquid Methals in a Rapidly Rotating Sphere," Phys. Rev. Lett. 119, 094501-1 (2017).

[3] K. Lam, D. Kong, and K. Zhang, "Nonlinear thermal inertial waves in rotating fluid spheres," Geophys. Astrophys. Fluid Dyn. 112, 357-374 (2018).

[4] Y. Lin, "Triadic resonances driven by thermal convection in a rotating sphere," J. Fluid Mech. 909, R3 (2021).

[5] K. Lam, D. Dali Kong, and K. Zhang, "Localised thermal convection in rotating spheres that undergo weak precession," Geophys. Astrophys. Fluid Dyn. 115, 280-296 (2021).

[6] S. Liu, Z.-H. Wan, R. Yan, C. Sun, and D.-J. Sun, "Onset of fully compressible convection in a rapidly rotating spherical shell," J. Fluid Mech. 873, 1090-1115 (2019).

[7] B. Wang, S. Liu, Z.-H. Wan, and D.-J. Sun, "Radius ratio dependency of the instability of fully compressible convection in rapidly rotating spherical shells," Journal of Fluid Mechanics 925, A40 (2021).

[8] T. T. Clarté, N. Schaeffer, S. Labrosse, and J. Vidal, "The effects of a robin boundary condition on thermal convection in a rotating spherical shell," Journal of Fluid Mechanics 918, A36 (2021).

[9] R. S. Long, J. E. Mound, C. J. Davies, and S. M. Tobias, "Scaling behaviour in spherical shell rotating convection with fixed-flux thermal boundary conditions," J.Fluid Mech. 889, A7-1-32 (2020).

[10] B. W. Hindman, N. A. Featherstone, and K. Julien, "Morphological classification of the convective regimes in rotating stars," Astrophys. J. 898, 120 (2020).

[11] R. K. Yadav, M. Heimpel, and J. Bloxham, "Deep convection-driven vortex formation on Jupiter and Saturn," Science Advances 6 (2020). 
[12] J. Sánchez, F. Garcia, and M. Net, "Critical torsional modes of convection in rotating fluid spheres at high Taylor numbers," J. Fluid Mech. 791, R1 (2016).

[13] K. Zhang, K. Lam, and D. Kong, "Asymptotic theory for torsional convection in rotating fluid spheres," J. Fluid Mech. 813, R2-1-R2-11 (2017).

[14] D. Kong, K. Zhang, K. Lam, and A. P. Willis, "Axially symmetric and latitudinally propagating nonlinear patterns in rotating spherical convection," Phys. Rev. E 98, 031101(R) (2018).

[15] J. Sánchez Umbría and M. Net, "Continuation of Double Hopf Points in Thermal Convection of Rotating Fluid Spheres," SIAM J. Appl. Dyn. Syst. 20, 208-231 (2021).

[16] J. Sánchez Umbría and M. Net, "Generation of bursting magnetic fields by nonperiodic torsional flows," Phys. Rev. E 100, 053110 (2019).

[17] J. Sánchez Umbría and M. Net, "Torsional solutions of convection in rotating fluid spheres," Phys. Rev. Fluids 4, 013501 (2019).

[18] K. Zhang, X. Liao, and P. Earnshaw, "On inertial waves and oscillations in a rapidly rotating fluid spheroid," J. Fluid Mech. 504, 1-40 (2004).

[19] D. J. Ivers, "Tilted incompressible Coriolis modes in spheroids," J. Fluid Mech. 833, 131-163 (2017).

[20] G. Backus and M. Rieutord, "Completeness of inertial modes of an incompressible inviscid fluid in a corotating ellipsoid," Phys. Rev. E 95, 053116, pp16 (2017).

[21] S. Maffei, A. Jackson, and P. W. Livermore, "Characterization of columnar inertial modes in rapidly rotating spheres and spheroids," Proc. Roy. Soc. Lond. A 473, 20170181 (2017).

[22] J. Rekier, S. A. Triana, A. Trinh, and V. Dehant, "Inertial Modes of a Freely Rotating Ellipsoidal Planet and Their Relation to Nutations," The Planetary Science Journal 1:20, 11pp (2020).

[23] K. H. Chan, X. Liao, and K. Zhang, "Simulations of fluid motion in spheroidal planetary cores driven by latitudinal libration." Phys. Earth Planet. Inter. 187, 404-415 (2011).

[24] K. Goto and R. A. van de Geijn, "Anatomy of high-performance matrix multiplication," ACM Trans. Math. Softw. 34, 1-25 (2008).

[25] B. Durney, "Convective Spherical Shell: II. With Rotation," J. Atmos. Sci. 25, 771-778 (1968).

[26] P. S. Marcus, "Stellar convection. I. Modal equations in spheres and spherical shells." Astrophys. J. 231, 176-192 (1979). 
[27] A. C. Hindmarsh, "ODEPACK, A Systematized Collection of ODE Solvers," IMACS Transactions on Scientific Computation, IMACS Transactions on Scientific Computation 1, 55-64 (1983).

[28] P. M. Mannix and A. J. Mestel, "Bistability and hysteresis of axisymmetric thermal convection between differentially rotating spheres," J. Fluid Mech. 911, A12 (2021).

[29] R. Simitev and F. H. Busse, "Patterns of convection in rotating spherical shells," New J. Phys. 5, 97.1-97.20 (2003).

[30] S. Chandrasekhar, Hydrodynamic and Hydromagnetic Stability (Oxford University Press, New York, 1961).

[31] M. Golubitsky, I. Stewart, and D. G. Schaeffer, Singularities and Groups in Bifurcation Theory, Vol. II (Springer, New York, 1988).

[32] C. Giberti and R. Zanasi, "Behavior of a three-torus in truncated Navier-Stokes equations," Physica D 65, 300-312 (1993).

[33] M. Net and J. Sánchez Umbría, "Periodic orbits in tall laterally heated rectangular cavities," Phys. Rev. E 95, 023102 (2017).

[34] J. Laskar, "Frequency analysis of a dynamical system," Celestial Mech. Dyn. Astr. 56, 191-196 (1993).

[35] J. M. Vega and S. Le Clainche, Higher Order Dynamic Mode Decomposition and Its Applications, 1st ed. (Academic Press, London, 2020).

[36] M. Sekikawa, N. Inaba, K. Kamiyama, and K. Aihara, "Three-dimensional tori and Arnold tongues," CHAOS 24, 013137 (2014).

[37] J. F. Yamagishi and K. Kaneko, "Chaos with a high-dimensional torus," Phys. Rev. Research 2, $023044(2020)$.

[38] H. Broer, C. Simó, and R. Vitolo, "Hopf saddle-node bifurcation for fixed points of 3ddiffeomorphisms: Analysis of a resonance 'bubble'," Physica D 237, 1773-1799 (2008).

[39] U. R. Christensen, "Zonal flow driven by deep convection in the major planets," Geophys. Res. Lett. 28 (2001).

[40] P. Cvitanović and J. F. Gibson, "Geometry of the turbulence in wall-bounded shear flows: periodic orbits," Physica Scripta T142, 014007 (2010).

[41] G. Kawahara, M. Uhlmann, and L. van Veen, "The Significance of Simple Invariant Solutions in Turbulent Flows," Ann. Rev. Fluid Mech. 44, 203-225 (2012). 